\title{
Correlations of light in the deep Fresnel region: An extended Van Cittert and Zernike theorem
}

\author{
Roberto Cerbino* \\ Dipartimento di Fisica, Università degli Studi di Milano, Via Celoria 16, I-20133 Milano, Italy
}

\begin{abstract}
We derive an extension of the Van Cittert and Zernike theorem (VCZT), which holds very near to planar quasihomogeneous radiation sources. This derivation is based on paraxial propagation of the Wigner distribution function of the scalar field. We identify a critical distance from the source. Below this distance (deep Fresnel region) the field autocorrelation function is invariant upon propagation and the propagated field contains information about the correlation properties of the radiation source. Beyond the critical distance, the classical VCZT theorem is found to hold and no information about the source correlation can be recovered. The transition between the two regimes is described analytically for a simple case.
\end{abstract}

The Van Cittert and Zernike theorem (VCZT) [1] is a milestone of modern optics. It describes the spatial coherence properties of radiation emitted from a planar incoherent source in terms of the two-dimensional (2D) Fourier transform (FT) of the source intensity distribution. In a classical formulation it can be stated as follows [2]. Given an extended incoherent light source at $z=0$ with intensity distribution $I_{0}(x, y)=\left|U_{0}(x, y)\right|^{2}$, the two-point field correlation function $J_{z}\left(x_{1}, y_{1} ; x_{2}, y_{2}\right)=\left\langle U_{z}\left(x_{1}, y_{1}\right) U_{z} *\left(x_{2}, y_{2}\right)\right\rangle$ (also called mutual intensity) at $z \neq 0$ is given by

$$
\begin{aligned}
J_{z}\left(x_{1}, y_{1} ; x_{2}, y_{2}\right) & =\frac{\kappa \exp \left[\frac{i 2 \pi(\bar{x} \Delta x+\bar{y} \Delta y)}{\lambda z}\right]}{\lambda^{2} z^{2}} \hat{I}_{0}\left(\frac{\Delta x}{\lambda z}, \frac{\Delta y}{\lambda z}\right) \\
& \doteq \kappa J_{z}^{\mathrm{VCZ}}(\bar{x}, \bar{y} ; \Delta x, \Delta y)
\end{aligned}
$$

where $\hat{I}_{0}(u, v)=\int I_{0}(x, y) e^{-i 2 \pi(u x+v y)} d x d y$ indicates the 2D FT of $I_{0}(x, y), \kappa$ is a constant with dimensions of squared length and, $\bar{x}=\left(x_{1}+x_{2}\right) / 2, \bar{y}=\left(y_{1}+y_{2}\right) / 2, \Delta x=x_{1}-x_{2}, \Delta y=y_{1}-y_{2}$. One of the important consequences of the VCZT is that a fully incoherent field can improve its coherence properties by free propagation in space.

This effect has been repeatedly used to measure the size of radiation sources of a different kind. In a very famous experiment performed in 1956, Hanbury-Brown and Twiss used a stellar intensity interferometer [3] and measured the diameter of Sirius by means of the VCZT. More recently the spatial coherence properties of synchrotron radiation have been deeply investigated by making use of the VCZT [4] and similar studies have been also performed on soft X-ray radiation obtained from noble gases by high order harmonic generation [5]. The VCZT has been also extensively used, both in reflection and transmission geometries, to describe the properties of the speckled intensity patterns caused by the interaction of light with rough surfaces [6]. Several instruments are based on this speckle effect, striking examples

\footnotetext{
*Present address: Physics Department, University of Fribourg, Ch. du Musée 3, CH-1700 Fribourg, Switzerland. Electronic address: cerbino@gmail.com
}

being the Electronic Speckle Pattern Interferometer (ESPI) and the Synthetic Aperture Radar (SAR).

The validity of the VCZT relies on some important assumptions. The paraxial or Fresnel approximation must hold i.e., the angles involved must be small. The source must be fully incoherent i.e., coherence cannot extend over more than a wavelength. From a mathematical point of view this last property is obtained by requiring that $J_{0}\left(x_{1}, y_{1} ; x_{2}, y_{2}\right)$ $=\kappa I_{0}(\bar{x}, \bar{y}) \delta(\Delta x, \Delta y)$ where $\delta(\cdot, \cdot)$ is the two dimensional $\delta$ function.

In 1965, a generalized Van Cittert and Zernike theorem (GVCZT) was provided by Goodman (see Ref. [2] for details) for the specific case of partially coherent sources described by a field correlation function of the form $J_{0}\left(x_{1}, y_{1} ; x_{2}, y_{2}\right)=I_{0}(\bar{x}, \bar{y}) \mu_{0}(\Delta x, \Delta y)$. Here the intensity $I_{0}$ is a slowly varying function with respect to the complex coherence factor $\mu_{0}$ (an equivalent statement is that the source linear size $D$ is large compared with the width $d$ of the complex coherence factor $\mu_{0}$ ). Following Carter and Wolf [7], we will refer to such kind of sources as planar quasihomogeneous sources, to stress that their second order correlation properties do not change across the illuminated region.

The GVCZT states that

$$
J_{z}\left(x_{1}, y_{1} ; x_{2}, y_{2}\right)=\frac{\kappa(\bar{x}, \bar{y}) \exp \left[\frac{i 2 \pi(\bar{x} \Delta x+\bar{y} \Delta y)}{\lambda z}\right]}{\lambda^{2} z^{2}} \hat{I}_{0}\left(\frac{\Delta x}{\lambda z}, \frac{\Delta y}{\lambda z}\right)
$$

which is formally identical to the VCZT provided that the constant $\kappa$ is replaced with the slowly varying function $\kappa(\bar{x}, \bar{y})=\hat{\mu}_{0}(\bar{x} / \lambda z, \bar{y} / \lambda z)$. The width of $\kappa(\bar{x}, \bar{y})$ is of the order $\lambda z / d$, much larger than the width of the function $\hat{I}_{0}(\Delta x / \lambda z, \Delta y / \lambda z)$ which is of the order $\lambda z / D$. Consequently, the effect of $\kappa(\bar{x}, \bar{y})$ on the correlation function is only a large scale modulation. It is worth pointing out that the GVCZT is derived in the paraxial approximation with the additional requirement that

$$
z \gg \frac{d D}{\lambda}
$$

and very recently [8] Gori showed that if condition (3) is fulfilled a generic source exhibits a VCZ-like behavior. 
What happens to the correlation function in the region $z$ $\ll d D / \lambda$ is still unknown.

In this paper we answer this question at least for quasihomogeneous sources. By using Wigner function propagation we derive a general expression for the propagated field correlation function. We also show that below the critical distance $z_{c}=d D / \lambda$ the propagated field correlation function coincides with the field correlation function on the source plane. For larger distances the classical predictions of the VCZ theorem are recovered. These results provide a full theoretical description of the findings of Giglio et al.[9] who demonstrated experimentally, for the case of speckle fields, the invariance of the intensity correlation function upon propagation.

The Wigner distribution function of a $2 \mathrm{D}$ signal $U_{z}(\mathbf{x})$ $=U_{z}(x, y)$ is defined [10] as

$$
W_{z}(\mathbf{x}, \mathbf{f})=\int U_{z}\left(\mathbf{x}+\frac{\mathbf{x}^{\prime}}{2}\right) U_{z}^{*}\left(\mathbf{x}-\frac{\mathbf{x}^{\prime}}{2}\right) e^{-i 2 \pi \mathbf{f} \cdot \mathbf{x}^{\prime}} d \mathbf{x}^{\prime}
$$

where $\mathbf{f}=\left(f_{x}, f_{y}\right)$. This distribution function represents the signal simultaneously in space and spatial frequencies and may therefore be seen as an intermediate representation of the true physical signal between the space $U(\mathbf{x})$ and the frequency $\hat{U}(\mathbf{f})$ representations. In the last $30 \mathrm{yrs}$ the concept of intermediate representations has been repeatedly used in optics and in this paper we will use it to describe the propagation of correlation functions of optical signals. As a starting point we can relate the Wigner distribution function to the signal correlation function with the Fourier transform relationship

$$
\left\langle W_{z}(\mathbf{x}, \mathbf{f})\right\rangle=\int J_{z}\left(\mathbf{x}+\frac{\mathbf{x}^{\prime}}{2} ; \mathbf{x}-\frac{\mathbf{x}^{\prime}}{2}\right) e^{-i 2 \pi \mathbf{f} \cdot \mathbf{x}^{\prime}} d \mathbf{x}^{\prime}
$$

which also implies

$$
J_{z}\left(\mathbf{x} ; \mathbf{x}^{\prime}\right)=J_{z}\left(\mathbf{x}+\frac{\mathbf{x}^{\prime}}{2} ; \mathbf{x}-\frac{\mathbf{x}^{\prime}}{2}\right)=\int\left\langle W_{z}(\mathbf{x}, \mathbf{f})\right\rangle e^{+i 2 \pi \mathbf{f} \cdot \mathbf{x}^{\prime}} d \mathbf{f} .
$$

Therefore a knowledge of the Wigner distribution function of the signal $U$ gives, by inverse Fourier transform, the signal correlation function. In the paraxial approximation, the Wigner distribution function changes by propagation according to the simple rule

$$
W_{z}(\mathbf{x}, \mathbf{f})=W_{0}(\mathbf{x}-\lambda z \mathbf{f}, \mathbf{f}) .
$$

Thus, with some calculation it is possible to derive an evolution equation for the correlation function

$$
J_{z}\left(\mathbf{x} ; \mathbf{x}^{\prime}\right)=\int d \mathbf{f} \int d \mathbf{x}^{\prime \prime} J_{0}\left(\mathbf{x}-\lambda z \mathbf{f} ; \mathbf{x}^{\prime \prime}\right) e^{-i 2 \pi \mathbf{f} \cdot\left(\mathbf{x}^{\prime \prime}-\mathbf{x}^{\prime}\right)}
$$

which is valid within the paraxial approximation for an arbitrary source.

By performing the identification $\mathbf{x}=\overline{\mathbf{x}}$ and $\mathbf{x}^{\prime}=\boldsymbol{\Delta} \mathbf{x}$ we can now specify Eq. (8) to quasihomogeneous sources. After some manipulation we obtain the simple result

$$
J_{z}(\overline{\mathbf{x}} ; \Delta \mathbf{x})=\mu_{0}(\Delta \mathbf{x}) \otimes J_{z}^{\mathrm{VCZ}}(\overline{\mathbf{x}} ; \boldsymbol{\Delta} \mathbf{x})
$$

i.e., the propagated field correlation function can be written as a convolution product between the complex coherence factor $\mu_{0}$ and the field correlation function predicted by the classical VCZT. It is worth noting that Eq. (9) could also be obtained with a direct calculation from the Huygens-Fresnel principle but the Wigner distribution function derivation followed here is very compact and elegant.

Equation (9) is the main result of this paper and its convolution form gives a very immediate feeling with the physics behind this problem. The width of the correlation function $\mu_{0}$ is approximately equal to $d$ and the width of $J_{z}^{\mathrm{VCZ}}$ is approximately $\lambda z / D$ (where $D$ is the source size). For $z$ $=d D / \lambda \doteq z_{c}$ the two functions have approximately the same width and we can identify two regimes:

(1) if $z \gg z_{c}$ the source act as a collection of $\delta$-correlated emitters i.e., $\mu_{0}(\Delta \mathbf{x}) \simeq \kappa \delta(\Delta \mathbf{x})$. Therefore the VCZT prediction of formula (1) is a good approximation for the propagated field correlation function.

(2) if $z \ll z_{c}$ the approximation $\hat{I}_{0}\left(\frac{\Delta \mathbf{x}}{\lambda z}\right) \simeq I_{0}(\overline{\mathbf{x}}) \delta\left(\frac{\Delta \mathbf{x}}{\lambda z}\right)$ $=\lambda^{2} z^{2} I_{0}(\overline{\mathbf{x}}) \delta(\boldsymbol{\Delta x})$ holds and we obtain

$$
J_{z}(\overline{\mathbf{x}} ; \Delta \mathbf{x}) \simeq I_{0}(\overline{\mathbf{x}}) \mu_{0}(\Delta \mathbf{x})=J_{0}(\overline{\mathbf{x}} ; \Delta \mathbf{x})
$$

i.e., the propagated field correlation function is approximately equal to that on the source plane $(z=0)$.

It is worth noting that in classical diffraction theory the transition from the Fresnel to the Fraunhofer regime (near to far field) for an aperture of size $D$ occurs at $z_{N F}=D^{2} / \lambda$. Therefore the validity condition for Eq. (10) is much more stringent than the usual near field condition because $d \ll D$. An equivalent statement is that Eq. (10) is valid for $N_{F}$ $\ll d / D \ll 1$ where $N_{F}=D^{2} / \lambda z$ is the Fresnel number of the illuminated aperture. According to Ref. [11] we will call this regime deep Fresnel region.

Due to the close analogy between classical coherence theory and speckle theory, all the above results are valid also for speckle fields provided that the concept of "planar quasihomogeneous source" is replaced by that of "rough object," i.e., a collection of independently-phased scatterers which, illuminated by a plane wave, act as a secondary source. This can be a rough surface illuminated in reflection geometry or a rough transmitting object such as a ground glass. As suggested in Ref. [9], a model object for this rough object is a random screen i.e., an opaque screen covered with randomly placed circular pinholes of size $d \gg \lambda$. This random screen has been used by the authors to show for the first time that, in the deep Fresnel regime, the size of the speckles (the width of the intensity autocorrelation function) does not change with distance. This property is a consequence of Eq. (9) if we take into account that the Siegert relation

$$
\left\langle I_{z}\left(\mathbf{x}_{1}\right) I_{z}\left(\mathbf{x}_{2}\right)\right\rangle=\left\langle I_{z}\right\rangle^{2}+\left|\left\langle U_{z}\left(\mathbf{x}_{1}\right) U_{z}^{*}\left(\mathbf{x}_{2}\right)\right\rangle\right|^{2}
$$

is valid [3]. This experimental regime, where speckle size does not change with distance has been also recently described by Goodman in a recent book [12]. 


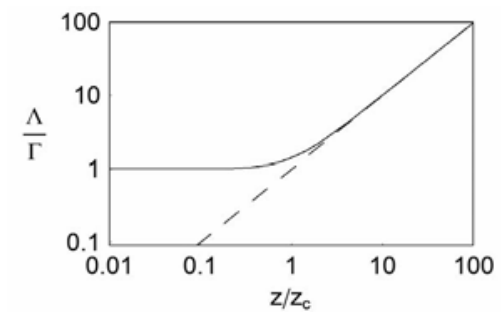

FIG. 1. The continuous line represents the normalized width of the field correlation function plotted as a function of the normalized distance from the source. For light sources this is the linear size of the correlation area. For speckle fields this is the linear size of a speckle. The dashed line is the far-field prediction obtained with the Van Cittert and Zernike theorem.

The last part of this paper is devoted to an analytical calculation for a simple model case. Let us start by considering a radiation source described by a Gaussian intensity profile of the form

$$
I_{0}(x, y)=I_{0}(0,0) \exp \left(-\frac{x^{2}+y^{2}}{2 \sigma^{2}}\right)
$$

and let us assume that the field on the source is described by a Gaussian correlation function

$$
\mu_{0}(\Delta x, \Delta y)=\exp \left(-\frac{\Delta x^{2}+\Delta y^{2}}{2 \Gamma^{2}}\right)
$$

By performing the convolution product in formula (9) we obtain

$$
\left|\left\langle U_{z}\left(\mathbf{x}_{1}\right) U_{z}^{*}\left(\mathbf{x}_{2}\right)\right\rangle\right| \propto \exp \left(-\frac{x^{2}+y^{2}}{2 \Lambda^{2}}\right)
$$

i.e., the propagated field correlation function is again Gaussian with width given by

$$
\Lambda(z)=\Gamma \sqrt{1+\left(\frac{\lambda z}{2 \pi \sigma \Gamma}\right)^{2}} .
$$

Equation (15) can be also rewritten in the following form

$$
\Lambda(z)=\Gamma \sqrt{1+\frac{z^{2}}{z_{c}^{2}}}
$$

where we have defined the critical distance as $z_{c}=2 \pi \Gamma \sigma / \lambda$ $=\pi d D / 4 \lambda$, and where $d$ and $D$ are respectively the $1 / e$ widths of $\mu_{0}(\Delta x, \Delta y)$ and $I_{0}(x, y)$. This result has a formal analogy with the formula describing the beam diameter of a Gaussian beam as a function of the distance from its beam waist, provided that the quantity $z_{c}$ is identified with the beam Rayleigh range [13].

In Fig. 1 we plot (continuous line) the width $\Lambda$ of the propagated field correlation function normalized with the width $\Gamma$ of the source field correlation function as a function of the ratio $z / z_{c}$. It appears clear that within the Rayleigh range one has $\Lambda=\Gamma$ but for larger distances one recovers exactly the VCZ like asymptotic behavior $\Lambda=\Gamma z / z_{c}$ $=\lambda z / 2 \pi \sigma=(\sqrt{2} / \pi) \lambda z / D$ (dashed line), with the correlation size increasing with distance $\mathrm{z}$.

An easy extension which represents a case of special interest for synchrotron people is that of a radiation source with an elliptical Gaussian intensity profile of the form

$$
I_{0}(x, y)=I_{0}(0,0) \exp \left(-\frac{x^{2}}{2 \sigma_{x}^{2}}-\frac{y^{2}}{2 \sigma_{y}^{2}}\right)
$$

but still described by a symmetric Gaussian correlation function such as in formula (13). An intensity distribution of this kind is a model for a source elongated along one direction such as for example a synchrotron x-ray insertion device (bending magnet or undulator). In this case we obtain

$$
\left|\left\langle U_{z}\left(\mathbf{x}_{1}\right) U_{z}^{*}\left(\mathbf{x}_{2}\right)\right\rangle\right| \propto \exp \left(-\frac{x^{2}}{2 \Lambda_{x}^{2}}-\frac{y^{2}}{2 \Lambda_{y}^{2}}\right)
$$

i.e., the propagated field correlation function is again an elliptical Gaussian but its size will be different along the vertical and horizontal directions according to

$$
\Lambda_{x, y}(z)=\Gamma \sqrt{1+\left(\frac{\lambda z}{2 \pi \sigma_{x, y} \Gamma}\right)^{2}} .
$$

If $\sigma_{x}>\sigma_{y}$ (i.e., source elongated horizontally such as a bending magnet $\mathrm{x}$-ray source) the Rayleigh range along the horizontal direction will be larger than the vertical one. This leads to a very peculiar situation: for very small distances the field correlation function will be circularly symmetric due to the circular symmetry of the correlation function of the field on the source plane. By moving at larger distances from the source, the correlation function will first deform in the vertical direction, still keeping the same horizontal size. To observe also a change in the horizontal direction one should measure the field correlation function at distances larger than the horizontal Rayleigh range.

In conclusion we have derived a generalization of a classical theorem in optics, i.e., the Van Cittert and Zernike theorem. In the deep Fresnel region of a quasihomogenous source, the field correlation function is found to be invariant upon propagation in striking agreement with recent experimental results. For larger distances the classical VCZ-like behavior is recovered and the correlation width becomes distance dependent. Some analytical results are also obtained.

The author is indebted to M. Giglio for many illuminating discussions. Fruitful discussions with D. Brogioli, M. Carpineti, A. Dogariu, D. Di Roberto, M.A.C. Potenza, and A. Vailati are also acknowledged. 
[1] P. H. Van Cittert, Physica (Utrecht) 1, 202 (1934); F. Zernike, Physica (Utrecht) 5, 785 (1938).

[2] J. W. Goodman, Statistical Optics (Wiley-Interscience, New York, 2000).

[3] R. Hanbury Brown and R. Q. Twiss, Nature (London) 178, 1046 (1956).

[4] J. J. A. Lin, D. Paterson, A. G. Peele, P. J. McMahon, C. T. Chantler, K. A. Nugent, B. Lai, N. Moldovan, Z. Cai, D. C. Mancini, and I. McNulty, Phys. Rev. Lett. 90, 074801 (2003).

[5] R. A. Bartels, A. Paul, H. Green, H. C. Kapteyn, M. M. Murnane, S. Backus, I. P. Christov, Y. Liu, D. Attwood, and C. Jacobsen, Science 297, 376 (2002).

[6] J. C. Dainty, ed., Laser Speckles and Related Phenomena (Topics in Applied Physics) (Springer-Verlag, Berlin, 1975).

[7] W. Carter and E. Wolf, J. Opt. Soc. Am. 67, 785 (1977).
[8] F. Gori, Opt. Lett. 30, 2840 (2005).

[9] M. Giglio, M. Carpineti, and A. Vailati, Phys. Rev. Lett. 85, 1416 (2000).

[10] M. J. Bastiaans, Application of the Wigner Function Distribution in Optics, in The Wigner Distribution-Theory and Applications in Signal Processing, edited by W. Mecklenbräuker and F. Hlawatsch (Elsevier Science, Amsterdam, Netherlands, 1997), pp. 375-426.

[11] S. Y. Teng, C. F. Cheng, M. Liu, W. L. Gui, and Z. Z. Xu, Chin. Phys. 14, 1990 (2005).

[12] J. W. Goodman, Speckle Phenomena in Optics (Roberts \& Company, Greenwood Village, CO, 2006), pp. 90-92.

[13] B. E. A. Saleh and M. C. Teich, Fundamentals of Photonics (Wiley-Interscience, New York, 1991). 